\title{
Gemcitabine and Cisplatin Combination Chemotherapy in Triple Negative Metastatic Breast Cancer Previously Treated with a Taxane/Anthracycline Chemotherapy; Multicenter Experience
}

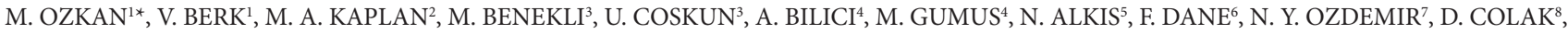 \\ M. DIKILITAS ${ }^{1}$
}

${ }^{1}$ Deparment of Medical Oncology, Erciyes University Medical School, Kayseri; ${ }^{2}$ Deparment of Medical Oncology, Dicle University Medical School, Diyarbakir; ${ }^{3}$ Deparment of Medical Oncology, Gazi University Medical School, Ankara; ${ }^{4}$ Deparment of Medical Oncology, Kartal Training and Research Hospital, İstanbul; ${ }^{5}$ Deparment of Medical Oncology, Ankara Oncology Hospital, Ankara; ${ }^{6}$ Deparment of Medical Oncology, Marmara University Medical School, İstanbul; ${ }^{7}$ Deparment of Medical Oncology, Ankara Numune Training and Research Hospital, Ankara; ${ }^{8}$ Deparment of Medical Oncology, Yildirim Beyazit Training and Research Hospital, Ankara

*Correspondence: metino@erciyes.edu.tr

Received June 30, 2011 / Accepted July 22, 2011

\begin{abstract}
This study was aimed to establish clinical efficacy and tolerability of gemcitabine and cisplatin combination in patients with metastatic triple negative breast cancer progressing after anthracycline and taxane based chemotherapies. Thirty-three patients who were given cisplatin and gemcitabine for triple negative and metastatic breast cancer were evaluated retrospectively. A total of 141 cycles were administered with a median 4 cycles per patient. Median follow-up time was 14 months (range, 2-36 months). Objective response rate was 27.3\%. Total clinical benefit of the combination was $48.4 \%$. The estimated median progression free survival and median overall survival were 5 months and 14 months, respectively. The most common Grade 3 and 4 toxicity were neutropenia and thrombocytopenia observed in $10(27.7 \%)$ and $9(24.9 \%)$ patients, respectively. The combination of the gemcitabine and cisplatin after taxane/anthracycline is well tolerated and seems to be effective with acceptable toxicity profile.
\end{abstract}

Key words: Triple negative, metastatic breast cancer, cisplatin, gemcitabine

Many factors are important in the management and prognosis of breast cancer (BC). These factors include patient characteristics (age, performance and menopausal status), tumor characteristics (histological grade, tumor size, hormone receptor status and expression of the human epidermal growth factor receptor 2[HER2]) and disease characteristics (stage, localization and disease free interval) (1). However, these clinico-pathological factors are insufficient to determine clinical response. Biological subtypes have been identified according to molecular markers depending on gene expression profiles and they were defined as: luminal-like A (estrogen receptors, ER-positive; progesterone receptors, PgR-positive and HER2-negative), luminal-like B (ER-positive, PgR-positive,

Abbreviations: ALT - Alanin aminotransferase, AST - Aspartate aminotransferase, $\mathrm{BC}$ - Breast cancer, $\mathrm{CR}$ - Complete response, PS - Performance status, SD - Stable disease, TNBC - Triple negative breast cancers
HER2-positive), basal-like (ER-negative, PgR-negative and HER2-negative), HER2-positive (ER-negative, PgR-negative and HER2-positive), and normal-like. Triple negative breast cancers (TNBCs) characterized by absence of ER, PgR and HER 2 expression and constitute $56-84 \%$ of basal-like tumors (2). Published in a recent study, TNBC patients constituted $70 \%$ of basal-like tumors (3). TNBC is associated with more aggressive clinical course and poor prognosis compared with the other subtypes. Many of the phenotypic and molecular features of TNBCs are similar to breast cancer with BRCA1 mutation.

Anthracyclines and taxanes are principal chemotherapeutic agents both in adjuvant and metastatic settings. No standard chemotherapy regimen has been proved to be effective in the treatment of anthracycline and taxane resistant TNBC. Therefore, there is an unmet need for an effective and safe salvage chemotherapy regimen. Gemcitabine is a nucleoside analogue 
that affects specific phases of the cell cycle. As a single agent, its objective response rate ranges between $29 \%-37 \%$ with a median time-to-progression of 5 to 8 months in metastatic breast cancer $(4,5)$.

Recent experimental data strongly suggest that cisplatin based chemotherapy could improve the outcome of triple-negative breast cancer (6). It is a bifunctional DNA cross-linking agent, leading to DNA damage. Therefore, DNA repair polymerases are activated. DNA repair polymerases uses gemcitabine for the repair and to begin signaling pathways leading to apoptosis (7). Gemcitabine and cisplatin combination have demonstrated synergy in in vivo and in vitro trials possible through synergistic interference tor DNA repair reported in several studies $(8,9)$.

This study was aimed to establish clinical efficacy and tolerability of gemcitabine and cisplatin combination in patients with metastatic TNBC progressing after anthracycline and taxane containing therapies.

\section{Material and methods}

Patients and procedure. Thirty-three patients with histologically proven, hormone receptor-negative and HER2negative metastatic breast cancer who received anthracycline and taxane containing chemotherapy for adjuvant or metastatic disease and then cisplatin and gemcitabine for TNBC were evaluated retrospectively. All patients had their specimens reviewed by standard immunohistochemistry methods in their central laboratories.

Women older than 18 years of age with measurable disease were included. Other inclusion criteria were as follows: Eastern Cooperative Oncology Group (ECOG) performance status (PS) 0-2, adequate liver (serum total bilirubin, aspartate aminotransferase (AST) and alanin aminotransferase (ALT) levels of $<$ twice the normal upper limit), bone marrow (white blood cells $>3 \times 10^{9} / 1$ or absolute neutrophil $>1.5 \times 10^{9} / 1$, platelets $>$ $100 \times 10^{9} / \mathrm{l}$, hemoglobin $>10 \mathrm{~g} / \mathrm{dl}$ ) and renal functions (serum creatinine level $<1.5 \mathrm{mg} / \mathrm{dl}$, blood urea nitrogen $<30 \mathrm{mg} / \mathrm{dl}$ ), creatinine clearance was required to exceed $60 \mathrm{ml} / \mathrm{min}$, no history of other malignancies. Patients with a history of brain metastases were allowed if they were asymptomatic.

Cisplatin $75 \mathrm{mg} / \mathrm{m}^{2}$ was given intravenously as an over 60 -min infusion on day 1 and Gemcitabine $1000 \mathrm{mg} / \mathrm{m}^{2}$ was administered intravenous infusion over $30 \mathrm{~min}$ on days 1 and 8 . The cycle has been repeated every 3 weeks. Doses of cisplatin and gemcitabine were reduced for grade 3-4 hematological and non-hematological toxicities. Toxicity was evaluated according to the Common Terminology Criteria for Adverse events v3.0 (CTCAE). Treatment was discontinued if disease progressed or unacceptable toxicity was observed.

Patients were evaluated every 2 cycles with physical examination and appropriate radiological and laboratory assessments. Complete response (CR) was defined as WHO response criteria disappearance of assessable disease and absence of disease-related symptoms. Partial response (PR) was defined as $>50 \%$ reduction in the product of the largest diameter target lesions without appearance of a new lesion for a duration of $>4$ weeks. Stable disease (SD) was defined as no change in tumor size or a $<25 \%$ increase or a $<25 \%$ decrease and for a duration of $>4$ weeks. Progressive disease (PD) was defined as an increase of more than $25 \%$ in tumor size or appearance a new lesion. Clinical benefit defined as the rate of $\mathrm{CR}+\mathrm{PR}+\mathrm{SD}>4$ weeks duration.

Statistical analysis. The overall survival (OS) time was calculated as the time from initiation of treatment to date of death or date of final follow-up examination. The progression-free survival (PFS) time was defined as from first day of treatment to clinical/radiological determination of progression or death. The Kaplan-Meier method was used for the survival analysis in SPSS 15.0 software program. Statistically significance was set at $\mathrm{p}<0.05$ level.

\section{Results}

Patient characteristics. Thirty-three eligible patients were enrolled between March 2007 and November 2009 from 8 oncology centers in Turkey. All patients were evaluated for toxicity, response, and survival. Patient characteristics are shown in Table 1. All patients had received anthracycline and taxane containing

Table1. Characteristics of the patients

\begin{tabular}{|c|c|}
\hline & $\begin{array}{c}\text { Patients }(\mathbf{n}=36) \\
\text { n (\%) }\end{array}$ \\
\hline Age (years) (Median) & 47 (Range, 27-73) \\
\hline \multicolumn{2}{|l|}{ Menopausal status } \\
\hline Premenopausal & $16(49)$ \\
\hline Postmenopausal & $17(51)$ \\
\hline \multicolumn{2}{|l|}{ Pathology } \\
\hline Invasive ductal & $28(85)$ \\
\hline Invasive lobular & $3(9)$ \\
\hline Mix and other & $2(6)$ \\
\hline \multicolumn{2}{|l|}{ Number of metastasis } \\
\hline Single & $14(42)$ \\
\hline Multiple & $19(58)$ \\
\hline \multicolumn{2}{|l|}{ Initial stage } \\
\hline Stage 2 & $10(30)$ \\
\hline Stage 3 & $17(52)$ \\
\hline Stage 4 & $6(18)$ \\
\hline \multicolumn{2}{|l|}{ Line of chemotherapy } \\
\hline First-line & $13(40)$ \\
\hline Second-line & $9(27)$ \\
\hline Third-line & $7(21)$ \\
\hline Fourth-line & $4(12)$ \\
\hline \multicolumn{2}{|l|}{ Family history } \\
\hline Yes & $2(6)$ \\
\hline No & $30(91)$ \\
\hline Unknown & $1(3)$ \\
\hline \multicolumn{2}{|l|}{ Performance status (ECOG) } \\
\hline 0 & $7(21)$ \\
\hline 1 & $23(70)$ \\
\hline 2 & $3(9)$ \\
\hline
\end{tabular}




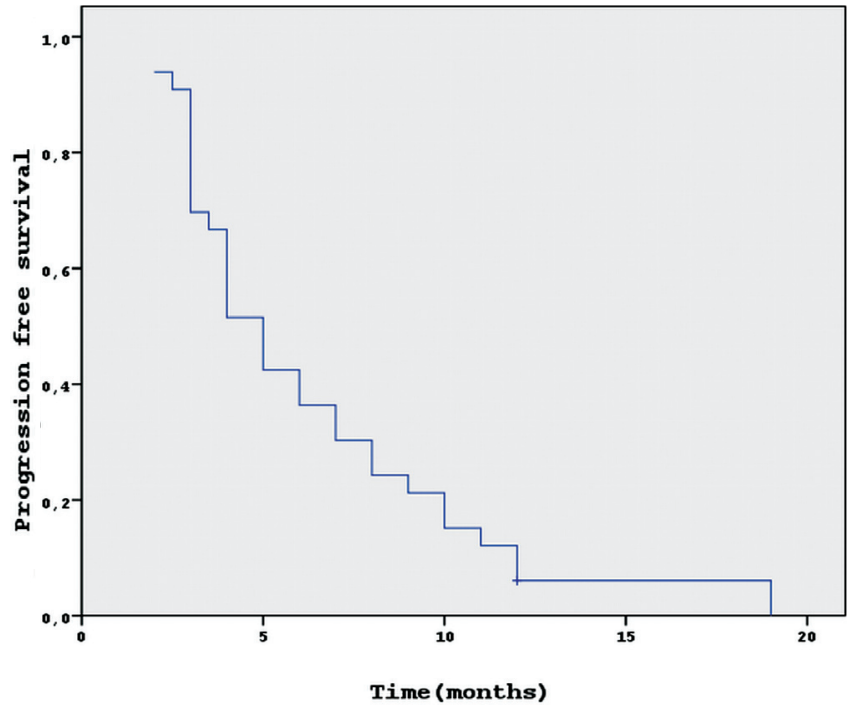

Figure 1. Progression free survival curve in metastatic TNBC patients received Cisplatin-Gemcitabine chemotherapy

chemotherapy regimen in metastatic or adjuvant setting. After adjuvant anthracycline and taxane, the median disease free survival was 17 months (range, 5-64) in the patients who were received gemcitabine-cisplatin combination metastatic first line. Median age was 47 years (range between 27 and 73 years). Approximately, $18 \%$ of patients had evidence of distant disease at the time of initial treatment; $82 \%$ of patients presented with early stage disease. The most common sites of metastasis were the bone (44\%), lung (38\%), liver (30\%) and brain (9\%).

Treatment and efficacy. A total of 141 cycles were administered and median number of cycles was 4 per patient (range, 2-8 cycles). Thirty-three patients were assessable for response. Of these patients, CR and PR rates were $6.1 \%$ and $21.2 \%$, respectively. Overall objective response rate was $27.3 \%$. SD and PD were observed in 7 (21.2\%) and 17 (51.5\%), respectively (Table 2). Total clinical benefit (objective response and stable disease) of the combination was $48.5 \%$. Median follow-up time was 14 months (range, 2-36 months) and 29 patients (88\%)

Table 2. Response to treatment and survival durations

\begin{tabular}{lc}
\hline Response of treatment & $\mathbf{n}(\%)$ \\
\hline Complete Response & $2(6.1)$ \\
Partial Response & $7(21.2)$ \\
Overall Response & $9(27.3)$ \\
Stable Disease & $7(21.2)$ \\
Progression & $17(51.5)$ \\
Clinical benefit of treatment & $16(48.5)$ \\
Survival Times & Months (95\% CI) \\
PFS & $5(3.9-6.0)$ \\
OS & $14(10.2-17.7)$ \\
\hline
\end{tabular}

PFS: progression free survival, OS: overall survival

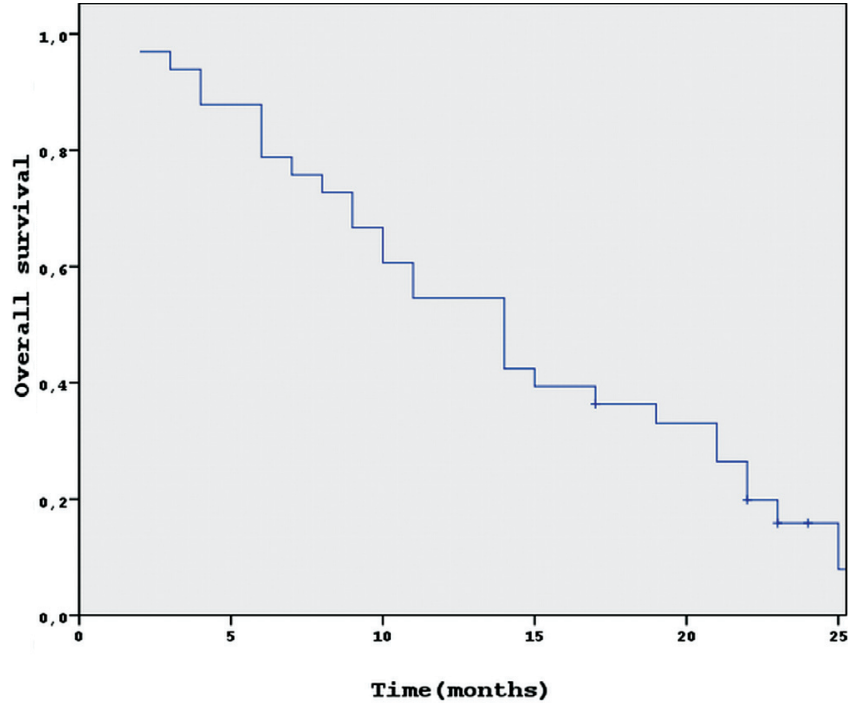

Figure 2. Overall survival curve in metastatic TNBC patients received Cisplatin-Gemcitabine chemotherapy

died at the time of last follow-up. Median response duration was 6.5 months (range, 2-13). Six-month and 1-year progression free survival rate was $35 \%$ and $8 \%$, while 6-month and 1 -year overall survival rate was $83 \%$ and $59 \%$, respectively. The estimated median progression free survival and median overall survival were 5 months (95\% CI, 3.95-6.04) and 14 months (95\% CI, 10.29-17.71), respectively (Figures 1 and 2). Out of three patients with brain metastases were not found significant difference in survival. There was no difference in respect to response rates between the patients having this combination or different lines of therapy.

Toxicities. Toxicities were evaluated in all 33 patients and the most common adverse event was neutropenia (61\%). Grade 3 and 4 neutropenia was observed in $10(27.7 \%)$ patients. Thrombocytopenia (all grade) was reported in 13 (36\%) patients including $5(13.8 \%)$ cases of grade 3 thrombocytopenia and $4(11.1 \%)$ cases of grade 4 thrombocytopenia. The most common Grade 3 and 4 non-hematologic toxicities included nausea and vomiting and were observed $13.8 \%$ of patients. Eleven patients (30\%) had developed grade 1 and 2 nausea and vomiting. Fatigue (all grade) was noted in $22 \%$ of patients. The other mild toxicities encountered were grade 1 anemia ( 6 patients) and grade 1 renal toxicities ( 2 patients). No chemotherapy-related death occurred. Doses of gemcitabine and Cisplatin were reduced by $20 \%$ during subsequent cycles when Grade 3 and 4 neutropenia and thrombocytopenia developed. Cisplatin doses were reduced by $20 \%$ in patients because of Grade 3 and 4 nausea and vomiting.

\section{Discussion}

TNBCs account for approximately $10-15 \%$ of all BCs and associated with poor survival compared to non-TNBC. 
Although anthracycline and taxane-containing regimens are favorable for treatment metastatic TNBCs, there is no standard first line chemotherapy regimen. The combination of cisplatin and gemcitabine has showed synergistic effects in in vitro trials. Inhibition of the repair of cisplatin induced DNA lesions plays a critical role in gemcitabine related cytotoxic synergism with cisplatin (10). Previously, efficacy and safety of this combination have been reported in both breast cancer and other cancer types including lung and bladder.

Various gemcitabine and cisplatin combination regimens have been evaluated in metastatic breast cancer. However, none of the studies reported any specific efficacy data for patients with TNBC. In a subgroup with aggressive clinical course such as TNBC, although similar or high response rates may be obtained, recurrences in short time intervals were observed as well (11). Our study demonstrated an objective response rate of $27.3 \%$, while the available studies have reported objective response rates ranging from $22.6 \%-54.5 \%$ in taxane and anthracycline resistant metastatic breast cancer. In the present study, observed median PFS and median OS were similar with the other studies. This observation reflects that cisplatin and gemcitabine combination is effective in TNBC as it is in other subgroup of patients and seems even to be superior in a group of selected patients (young, symptomatic, having adequate bone marrow capacity). There are several clinical trails in metastatic TNBCs. Uhm et al. (12) have used taxane plus platinum combination chemotherapy in patients with metastatic TNBC. Objective response was obtained in nine of 24 patients (37.5\%). The median PFS and OS were 6.3 and 19.3 months, respectively. Yi et al. (13) reported a response rate of $38.8 \%$ with cisplatin containing regimens in metastatic TNBC. Chia et al. (14) observed a clinical benefit in 10 (71\%) of 14 patients with paclitaxel and carboplatin combination. Thome et al. (15) evaluated a combination of gemcitabine and erlotinib in metastatic TNBC. While 5 of 20 patients (25\%) had clinical benefit, median PFS and OS were 72 and 227 days, respectively.

As there are only few studies with small number of patients conducted in TBNC evaluating efficacy of platinum containing combinations, large scaled trials are warranted. Our study is consistent with the literature in respect to survival results. Having relatively larger number of patients compared to other studies reported previously our study has also limitations as it is a retrospective multicenter study. TNBCs and BRCA1-mutated breast cancers present similar molecular, pathologic and clinical behavior. BRCA1 inhibits apoptosis and regulates mitosis after treatment with agents which are DNA-damaging (16). PARP1 is activated by DNA damage and it binds to DNA strand break for repairing (17). PARP1 inhibitors reduce survival function in BRCA1-deficient cells. Combination of the PARP1 inhibitors with platinum agents in the BRCA1-deficient cells mouse mammary tumor increased the survival time (18). O'Shaughnessy et al. (19) reported that a clinical benefit rate of $62 \%$ with combination of carboplatin plus gemcitabine and PARP1 inhibitors in the TNBC. This group had significantly prolonged median PFS of 6.9 months compared with 3.3 months carboplatin and gemcitabine alone group. Median OS was significantly shorter for carboplatin and gemcitabine alone group: 5.7 versus 9.2 months. This was the largest phase II randomized clinical trial and it is remarkable that survival time with carboplatin gemcitabine combination was quite shorter than reported in other studies and also in ours. And also as a result of overlapping hematologic toxicity of carboplatin and gemcitabine, grade 3 or 4 hematologic toxicity was found very high. This combination therapy didn't show same efficacy in Phase III trial. High risk hematologic toxicities of PARP inhibitor in combination with the chemotherapy regimen was the controversial point (20). In the light of the potential effects and side effects of this combination, we suggest using cisplatin instead of carboplatin.

In the present study, gemcitabine and cisplatin combination related toxicities were acceptable. Grade 3 and 4 neutropenia and thrombocytopenia was observed in $27.7 \%$ and $24.9 \%$, respectively. Hematologic side-effects were acceptable and similar with the other trails. Nausea and vomiting was frequently seen as non-hematologic toxicities. Only 1 patient discontinued therapy for grade 4 nausea and vomiting. Since aprepitant was used, it has been very successful preventing cisplatin related nausea and vomiting.

In summary, a convenient regimen for the salvage treatment of anthracycline and taxane pretreated metastatic TNBC has yet not been determined. Combination of the gemcitabine and cisplatin is well tolerated and seems to be effective. This combination is worthy to evaluate in early steps in the treatment of TNBC and also with novel therapeutic agents such as anti-angiogenics, EGFR, PARP1 inhibitors.

\section{References}

[1] National Comprehensive Cancer Network. NCCN Practice Guidelines in Oncology- v.1.2009: Breast Cancer. http://www. nccn.org/clinical.asp. Accessed November 2009

[2] SASAKI Y, TSUDA H Clinicopathological characteristics of triple-negative breast cancers. Breast Cancer 2009; 16: 254259. http://dx.doi.org/10.1007/s12282-009-0153-5

[3] DOGU GG, OZKAN M, OZTURK F, DIKILITAS M, ER O et al. Triple-negative breast cancer: immunohistochemical correlation with basaloid markers and prognostic value of survivin. Med Oncol. 2010; 27: 34-9. http://dx.doi.org/10.1007/s12032009-9166-3

[4] SPIELMANN M, LLOMBART-CUSSAC A, KALLA S, ESPIE $M$, NAMER $M$ et al. Single-agent gemcitabine is active in previously treated metastatic breast cancer. Oncology 2001; 60:3 03-307. http://dx.doi.org/10.1159/000058524

[5] BLACKSTEIN M, VOGEL CL, AMBINDER R, COWAN J, IGLESIAS J et al. Gemcitabine as first-line therapy in patients with metastatic breast cancer: a phase II trial. Oncology 2002; 62: 2-8. http://dx.doi.org/10.1159/000048240

[6] SILVER DP, RICHARDSON AL, EKLUND AC, WANG ZC, SZALLASI Z et al. Efficacy of neoadjuvant Cisplatin in triple- 
negative breast cancer. J Clin Oncol. 2010; 28(7): 1145-53. http://dx.doi.org/10.1200/JCO.2009.22.4725

[7] ACHANTA G, PELICANO H, FENG L, PLUNKETT W, HUANG P Interaction of p53 and DNA-PK in response to nucleoside analogues: potential role as a sensor complex for DNA damage. Cancer Res 2001; 61: 8723-8729.

[8] PETERS GJ, BERGMAN AM, RUIZ VAN HAPEREN VW, VEERMAN G, KUIPER CM et al. Interaction between cisplatin and gemcitabine in vitro and in vivo. Semin Oncol 1995; 22 (4 Suppl 11): 72-79.

[9] HEINEMANN V, STEMMLER HJ, WOHLRAB A, BOSSE D, LOSEM C et al. High efficacy of gemcitabine and cisplatin in patients with predominantly anthracycline- and taxane-pretreated metastatic breast cancer. Cancer Chemother Pharmacol 2006; 57: 640-646. http://dx.doi.org/10.1007/s00280-005$\underline{0093-5}$

[10] GIOVANNETTI E, DANESI R, MEY V, NANNIZZI S, PASQUALETTI $G$ et al. In vitro studies on gemcitabine combinations with other antiblastics. Ann Oncol 2006; 17 (Suppl 5): v17-19. http://dx.doi.org/10.1093/annonc/mdj943

[11] JASPERS JE, ROTTENBERG S, JONKERS J Therapeutic options for triple-negative breast cancers with defective homologous recombination. Biochim Biophys Acta 2009; 1796: 266-280.

[12] UHM JE, PARK YH, YI SY, CHO EY, CHOI YL et al. Treatment outcomes and clinicopathologic characteristics of triple-negative breast cancer patients who received platinumcontaining chemotherapy. Int J Cancer 2009; 124: 1457-1462. http://dx.doi.org/10.1002/ijc. 24090

[13] YI S, UHM J, CHO E, LEE S, PARK M et al. Program and abstracts of the 2008 Annual Meeting of the American Society of Clinical Oncology; abstract 1008.
[14] CHIA J.W, ANG P, SEE H, WONG Z, SOH L et al. Program and abstracts of the 2007 Annual Meeting of the American Society of Clinical Oncology; abstract 1086.

[15] THOME S, HOBDAY T, HILLMAN D, AUBRY M, LINGLE W et al. Program and abstracts of the 2007 Annual Meeting of the American Society of Clinical Oncology; abstract 1071.

[16] HUSAIN A, HE G, VENKATRAMAN ES, SPRIGGS DR BRCA1 up-regulation is associated with repair-mediated resistance to cis-diamminedichloroplatinum (II). Cancer Res 1998; 15: 1120-1123.

[17] SCHREIBER V, DANTZER F, AME JC, DE MURCIA G. Poly (ADP-ribose): novel functions for an old molecule. Nat Rev Mol Cell Biol 2006; 7: 517-528. http://dx.doi.org/10.1038/nrm1963

[18] ROTTENBERG S, JASPERS JE, KERSBERGEN A, VAN DER BURG E, NYGREN AO et al. High sensitivity of BRCA1deficient mammary tumors to the PARP inhibitor AZD2281 alone and in combination with platinum drugs. Proc Natl Acad Sci USA 2008; 105: 17079-84. http://dx.doi.org/10.1073/ pnas.0806092105

[19] O SHAUGHNESSY J, OSBORNE C, PIPPEN J, YOFFE M, PATT D et al. Final Results of a Randomized Phase II Study Demonstrating Efficacy and Safety of BSI-201, a Poly (ADPRibose) Polymerase (PARP) Inhibitor, in Combination with Gemcitabine/Carboplatin (G/C) in Metastatic Triple Negative Breast Cancer (TNBC). San Antonio Breast Cancer Symposium December 9-13, 2009 San Antonio, Texas, USA. Abstract 3122.

[20] J. O'SHAUGHNESSY, L. S. SCHWARTZBERG, M. A. DANSO, H. S. RUGO, K. MILLER et al. A randomized phase III study of iniparib (BSI-201) in combination with gemcitabine/carboplatin $(\mathrm{G} / \mathrm{C})$ in metastatic triple-negative breast cancer (TNBC). Program and abstracts of the 2011 Annual Meeting of the American Society of Clinical Oncology. Abstract No: 1007 